\title{
AN EVOLUTIONARY PROGRAMMING BASED TABU SEARCH METHOD FOR UNIT COMMITMENT PROBLEM WITH COOLING-BANKING CONSTRAINTS
}

\author{
C. Christober Asir Rajan
}

\begin{abstract}
This paper presents a new approach to solve the short-term unit commitment problem using An Evolutionary Programming Based tabu search method with cooling and banking constraints. Numerical results are shown comparing the cost solutions and computation time obtained by using the evolutionary programming method and other conventional methods like dynamic programming, lagrangian relaxation.
\end{abstract}

K e y w ords: unit commitment problem, tabu search, evolutionary programming

\section{INTRODUCTION}

Unit commitment is the problem of selecting the generating units to be put into service during a scheduling period and for how long. The committed units must meet the system load and reserve requirements at minimum operating cost, subject to a variety of constraints. Unit commitment is an important optimization task in the daily operating planning of power system. The solution of unit commitment problem (UCP) is really a complex optimization problem. It can be considered as two linked optimization problems, the first is a combinatorial problem of generating units, which could be a very huge number. TS is a powerful optimization procedure that has been successfully applied to a number of combinatorial optimization problems. It has the ability to avoid entrapment in local minima. TS employ a flexible memory system (in contrast to 'memory less' systems, such as simulated annealing and genetic algorithm, and rigid memory system such as in branch-and-bound). Specific attention is given to the short-term memory component of TS, which has provided solutions superior to the best obtained with other methods for a variety of problems.

Research endeavours, therefore, have been focused on; efficient, near-optimal UC algorithms, which can be applied to large-scale, power systems and have reasonable storage and computation time requirements. A survey of existing literature [1-26] on the problem reveals that various numerical optimisation techniques have been employed to approach the complicated unit commitment problem. More specifically, these are the dynamic programming method (DP), the mixed integer programming method (MIP), the lagrangian relaxation method (LR), the branch and bound method $(\mathrm{BB})$, the expert system (ES), the fuzzy theorem method (FT), the hop field method $(\mathrm{H})$, the simulated annealing method (SA), the tabu search (TS), the genetic algorithm (GA), the artificial neural network (ANN), the evolutionary programming (EP) and so on. The major limitations of the numerical techniques are the problem dimensions, large computational time and complexity in programming.
The DP method [1-2], [13] is flexible but the disadvantage is the "curse of dimensionality", which results it may leads to more mathematical complexity and increase in computation time if the constraints are taken in to consideration. The MIP methods [3-4] for solving the unit commitment problems fail when the number of units increases because they require a large memory and suffer from great computational delay. The LR approach [58] to solve the short-term UC Problems was found that it provides faster solution but it will fail to obtain solution feasibility and solution quality problems and becomes complex if the number of units increased. The BB method [9] employs a linear function to represent fuel cost and start-up cost and obtains a lower and upper bounds. The difficulty of this method is the exponential growth in the execution time for systems of a practical size. An ES algorithm [10], [13] rectifies the complexity in calculations and saving in computation time. But it will face the problem if the new schedule is differing from schedule in database. In the FT method [11], [13], [24] using fuzzy set solves the forecasted load schedules error but it will also suffer from complexity. The $\mathrm{H}$ neural network technique [12] considers more constraints but it may suffer from numerical convergence due to its training process. SA [14-17], [23-24] is a powerful, general-purpose stochastic optimisation technique, which can theoretically converge asymptotically to a global optimum solution with probability one. But it will take much time to reach the near-global minimum. The TS [18-20], [23] is an iterative improvement procedure that starts from some initial feasible solution and attempts to determine a better solution in the manner of a greatest-decent algorithm. However, TS is characterized by an ability to escape local optima by using a short-term memory of recent solutions.

GA [13], [21-24] is a general-purpose stochastic and parallel search method based on the mechanics of natural selection and natural genetics. It is a search method to have potential of obtaining near-global minimum. And it has the capability to obtain the accurate results within short time and the constraints are included easily. The

* Department of EEE Pondicherry Engineering College Pondicherry - 605014 India, asir_70@hotmail.com 
ANN [12], [28] has the advantages of giving good solution quality and rapid convergence. And this method can accommodate more complicated unit-wise constraints and are claimed for numerical convergence and solution quality problems. The solution processing in each method is very unique. The EP [25-26] has the advantages of good convergent property and a significant speedup over traditional GA's and can obtain high quality solutions. The "curse of dimensionality" is surmounted, and the computational burden is almost linear with the problem scale.

From the literature review, it has been observed that there exists a need for evolving simple and effective methods, for obtaining an optimal solution for the UCP. Hence, in this paper, an attempt has been made to couple EP with TS for meeting these requirements of the UCP, which eliminates the above-mentioned drawbacks. In case of TS, the demand is taken as control parameter. Hence the quality of solution is improved. The algorithm is based on the annealing neural network. Classical optimization methods are a direct means for solving this problem. EP seems to be promising and is still evolving. EP has the great advantage of good convergent property and, hence, the computation time is considerably reduced. The EP combines good solution quality for TS with rapid convergence for EP. The EP Based TS (EPTS) is used to find the short- term thermal unit commitment. By doing so, it can help to find the optimum solution rapidly and efficiently.

EP is capable of determining the global or near global solution. It is based on the basic genetic operation of human chromosomes. It operates with the stochastic mechanics, which combine offspring creation based on the performance of current trail solutions and competition and selection based on the successive generations, form a considerably robust scheme for large-scale real-valued combinational optimization. In this proposed work, the parents are obtained from a pre-defined set of solution's $i e$ each and every solution is adjusted to meet the requirements. And the selection process is done using Evolutionary Strategy [8-9].

\section{PROBLEM FORMULATION}

The objective is to find the generation scheduling such that the total operating cost can be minimized, when subjected to a variety of constraints [27]. In the UCP under consideration, an interesting solution would be minimizing the total operating cost of the generating units with several constraints being satisfied. The major component of the operating cost, for thermal and nuclear units, is the power production cost of the committed units and this is given in a quadratic form

$$
F_{i t}\left(P_{i t}\right)=A_{i} P_{i t}^{2} B_{i} P_{i t}+C_{i} \mathrm{Rs} / \mathrm{hr},
$$

where, $A_{i}, B_{i}, C_{i}$ - are the cost function parameters of unit $i,\left(\mathrm{Rs} / \mathrm{MW}^{2} \mathrm{hr}, \mathrm{Rs} / \mathrm{MWhr}, \mathrm{Rs} / \mathrm{hr}\right), F_{i t}\left(P_{i t}\right)$ - is the production cost of unit $i$ at a time $t(\mathrm{Rs} / \mathrm{hr}), P_{i t}-$ is the output power from unit $i$ at time $t$ (MW).
The start up cost depends upon the down time of the unit, which can vary from a maximum value, when the unit $\mathrm{i}$ is started from cold state, to a much smaller value, if the unit i has been turned off recently. The start up cost calculation depends upon the treatment method for the thermal unit during down time periods. The start-up cost $S_{i t}$, is a function of the down time of unit $i$ given as

$$
S_{i t}=S o_{i}\left[1-D_{i} \exp \left(-\operatorname{Toff}_{i} / \operatorname{Tdown}_{i}\right)\right]+E_{i}
$$

where, $S o_{i}$ - is the unit $i$ cold start-up cost (Rs), $D_{i}, E_{i}$ - are the start-up cost coefficients for unit $i$.

The overall objective function of the UCP is then

$$
F_{T}=\sum_{t=1}^{T} \sum_{i=1}^{N}\left(F_{i t}\left(P_{i t}\right) U_{i t}+S_{i t} V_{i t}\right)
$$

where, $U_{i t}$ - is the unit $i$ status at hour $t=1$ (if unit is $\mathrm{ON})=0$ (if unit is OFF), $V_{i t}$ - is the unit $i$ start up/shut down status at hour $t=1$ if the unit is started at hour $t$ and 0 otherwise, $F_{T}$ - is the total operating cost over the schedule horizon $(\mathrm{Rs} / \mathrm{hr}), S_{i t}$ - is the start up cost of unit $i$ at hour $t$ (Rs).

\subsection{Constraints}

Depending on the nature of the power system under study, the UCP is subject to many constraints, the main being the load balance constraints and the spinning reserve constraints. The other constraints include the thermal constraints, fuel constraints, security constraints etc [27].

\subsubsection{Load Balance Constraints}

The real power generated must be sufficient enough to meet the load demand and must satisfy the following factors

$$
\sum_{i=1}^{N} P_{i t} U_{i t}=P D_{t}
$$

where, $P D_{t}$ - is the system peak demand at hour $t$ (MW), $N$ - is the number of available generating units, $U(0,1)$ - is the uniform distribution with parameters 0 and $1, U D(a, b)$ - is the discrete uniform distribution with parameters $a$ and $b$.

\subsubsection{Spinning Reserve Constraints}

The spinning reserve is the total amount of real power generation available from all synchronized units minus the present load plus the losses. The reserve is considered to be a pre specified amount or a given percentage of the forecasted peak demand. It must be sufficient enough to meet the loss of the most heavily loaded unit in the system. This has to satisfy the equation given in (5).

$$
\sum_{i=1}^{N} P \max _{i} U_{i t} \geq\left(P D_{t}+R_{t}\right) ; 1 \leq t \leq T,
$$

where, $P \max _{i}$ - is the maximum generation limit of unit $i, R_{t}$ - is the spinning reserve at time $t(\mathrm{MW}), T-$ is the scheduled time horizon $(24 \mathrm{hr})$. 


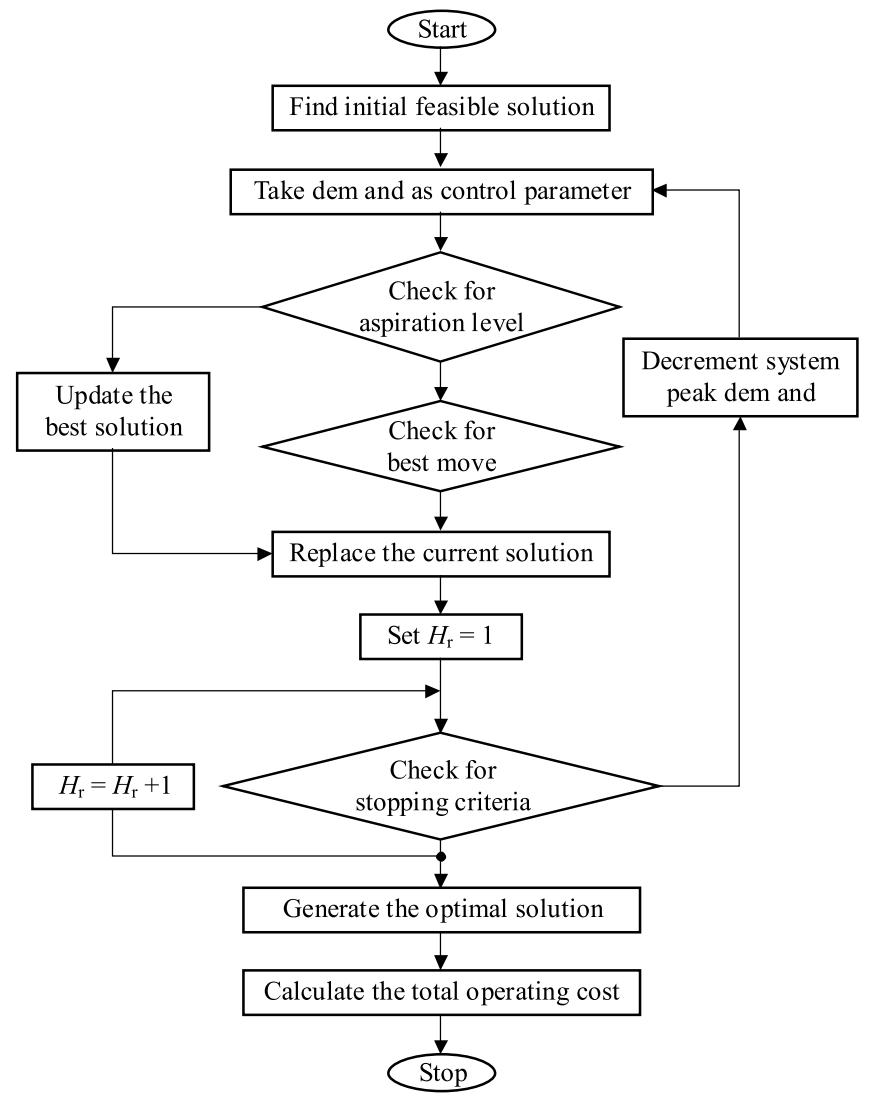

Fig. 1. Flowchart of Tabu Search Algorithm

\subsubsection{Thermal Constraints}

The temperature and pressure of the thermal units vary very gradually and the units must be synchronized before they are brought online. A time period of even 1 hour is considered as the minimum down time of the units. There are certain factors, which govern the thermal constraints, like minimum up time, minimum down time and crew constraints.

\section{a) Minimum up time}

If the units have already been shut down, there will be a minimum time before they can be restarted and the constraint is given in (6).

$$
\text { Ton }_{i}=\text { Tup }_{i}
$$

where $\operatorname{Ton}_{i}$ - is the duration for which unit $i$ is continuously $\mathrm{ON}(\mathrm{hr}), \mathrm{Tup}_{i}$ - is the unit $i$ minimum up time (hr).

\section{b) Minimum down time}

If all the units are running already, they cannot be shut down simultaneously and the constraint is given in (7).

$$
\text { Toff }_{i} \geq \text { Tdown }_{i},
$$

where, Tdown $_{i}$ - unit $i$ minimum down time (hr), Toff ${ }_{i}^{-}$ duration for which unit $i$ is continuously OFF (hr).

\subsubsection{Must Run Units}

Generally in a power system, some of the units are given a must run status in order to provide voltage support for the network.

\section{TABU SEARCH}

\subsection{Overview}

In solving the UCP, two types of variables need to be determined. The unit's status variables $U$ and $V$, which are integer variables and the units, output power variables $P$ that are continuous variables. The problem can then be decomposed into two sub problems, a combinatorial problem in $U$ and $V$ and a non-linear optimization problem in $P$. TS are used to solve the combinatorial optimization and the non-linear optimization is solved via a quadratic programming routine [18]. The flowchart for TS is shown in Fig. 1.

The proposed algorithm contains three major steps:

- First, generating randomly feasible trail solutions.

- Second, calculating the objective function of the given solution by solving the EDP.

- Third, applying the TS procedures to accept or reject the solution in hand.

\subsection{Tabu Search General Algorithm}

Step 0: Assume that the fuel costs to be fixed for each hour and all the generators share the loads equally.

Step 1: By optimum allocation find the initial feasible solution $\left(U_{i}, V_{i}\right)$.

Step 2: Demand is taken as the control parameter.

Step 3: Generate the trial solution.

Step 4: Calculate the total operating cost, $F_{t}$, as the summation of running cost and Start up-shut down cost.

Step 5: Tabulate the fuel cost for each unit for every hour.

\subsection{Generating Trial Solution}

The neighbors should be randomly generated, feasible, and span as much as possible the problem solution space. Because of the constraints in the UCP this is not a simple matter. The most difficult constraints to satisfy are the minimum up/down times. The implementation of new rules to obtain randomly feasible solutions faster are done by the rules is described in [18].

\subsection{Generating an Initial Solution}

The TS algorithm requires a starting feasible schedule, which satisfies all the system and units constraints. This schedule is randomly generated. The algorithm given in [18] is used for finding this starting solution.

\subsection{Operating Cost Calculation}

Once a trail solution is obtained, the corresponding total operating cost is determined. Since the production cost is a quadratic function, the EDP is solved using a quadratic programming routine. The start-up cost is then calculated for the given schedule. 


\subsection{Generating an Initial Solution}

The TS algorithm requires a starting feasible schedule, which satisfies all the system and units constraints. This schedule is randomly generated. The algorithm given in [18] is used for finding this starting solution.

\subsection{Operating Cost Calculation}

Once a trail solution is obtained, the corresponding total operating cost is determined. Since the production cost is a quadratic function, the EDP is solved using a quadratic programming routine. The start-up cost is then calculated for the given schedule.

\subsection{Stopping Criteria}

There may be several stopping criteria for the search. For this implementation, the search is stopped if the following conditions are satisfied:

- The load balance constraints are satisfied.

- The spinning reserve constraints are satisfied.

\section{EVOLUTIONARY PROGRAMMING}

\subsection{Introduction}

EP is a mutation-based evolutionary algorithm applied to discrete search spaces. David Fogel [29] extended the initial work of his father Larry Fogel [31] for applications involving real-parameter optimization problems. Real-parameter EP is similar in principle to evolution strategy (ES), in that normally distributed mutations are performed in both algorithms. Both algorithms encode mutation strength (or variance of the normal distribution) for each decision variable and a self-adapting rule is used to update the mutation strengths. Several variants of EP have been suggested [29].

\subsection{Evolutionary Strategies}

For the case of Evolutionary strategies D.B. Fogel remarks "evolution can be categorized by several levels of hierarchy: the gene, the chromosome, the individual, the species, and the ecosystem." Thus, while Genetic Algorithms stress models of genetic operators, Evolutionary Strategies emphasize mutational transformation that maintains behavioral linkage between each parent and its offspring at the level of the individual. Evolutionary Strategies are a joint development of Bienert, Rechenberg, and Schwefel. The first applications were experimental and addressed some optimization problems in hydrodynamics.

\subsection{EP General Algorithm}

Evolutionary programming [25-26], [29-31] is conducted as a sequence of operations and is given below.

1. The initial population is determined by setting $s_{i}=$ $S_{i} \sim U\left(a_{k}, b_{k}\right)^{k} i=1, \ldots, m$, where $S_{i}$ is a random vector, $s_{i}$ is the outcome of the random vector, $U\left(a_{k}, b_{k}\right)^{k}$ denotes a uniform distribution ranging over $\left[a_{k}, b_{k}\right]$ in each of $k$ dimensions, and $m$ is the number of parents.
2. Each $s_{i}, i=1, \ldots, m$, is assigned a fitness score $\vartheta\left(s_{i}\right)=G\left(F\left(s_{i}\right), v_{i}\right)$, where $F$ maps $s_{i} \mapsto R$ and denotes the true fitness of $s_{i}, v_{i}$, represents random alteration in the instantiation of $s_{i}$, random variation imposed on the evaluation of $F\left(s_{i}\right)$, or satisfies another relation $s_{i}$, and $G\left(F\left(s_{i}\right), v_{i}\right)$ describes the fitness score to be assigned. In general, the functions $F$ and $G$ can be as complex as required. For example, $F$ may be a function not only of a particular $s_{i}$, but also of other members of the population, conditioned on a particular $s_{i}$.

3. Each $s_{i}, i=1, \ldots, m$, is altered and assigned to $s_{i+m}$ such that $s_{i+m}=s_{i, j}+N\left(0, \beta_{j} \vartheta\left(s_{i}\right)+z_{j}\right), j=1, \ldots, k$. $N\left(0, \beta_{j} \vartheta\left(s_{i}\right)+z_{j}\right)$ represents a Gaussian random variable with mean $\mu$ and variance $\sigma^{2}, \beta_{j}$ is a constant of proportionality to scale $\vartheta\left(s_{i}\right)$, and $z_{j}$ represents an offset to guarantee a minimum amount of variance,

4. Each $s_{i+m}, i=1, \ldots, m$, is assigned a fitness score $\vartheta\left(s_{i+m}\right)=G\left(F\left(s_{i+m}\right), v_{i+m}\right)$

5. For each $s_{i}, i=1, \ldots, 2 m$, a value $w_{i}$ is assigned according to

$$
w_{i}=\sum_{t=1}^{c} w_{t}^{*}, \quad w_{t}^{*}= \begin{cases}1 & \text { if } \vartheta\left(s_{i}\right) \leq \vartheta\left(s_{t}\right) \\ 0, & \text { otherwise }\end{cases}
$$

where $p=\left[2 m u_{1}+1\right], p \neq i,[x]$ denotes the greatest integer less than or equal to $x, c$ is the number of competitions, and $u_{1} \sim U(0,1)$.

6 . The solutions $s_{i}, i=1 \ldots, 2 m$, are ranked in descending order of their corresponding value $w_{i}$ [with preference to their actual scores $\vartheta\left(s_{i}\right)$ if there are more than $m$ solutions attaining a value of $c]$. The first $m$ solutions are transcribed along with their corresponding values $\vartheta\left(s_{i}\right)$ to be the basis of the next generation.

7. The process proceeds to step 3 , unless the available execution time is exhausted or an acceptable solution has been discovered.

\subsection{Evolutionary Programming for UCP}

1. Initialize the parent vector $p=\left[p_{1}, p_{2}, \ldots, p_{n}\right], i=$ $1,2, \ldots, N_{p}$ such that each element in the vector is determined by $p_{j} \sim \operatorname{random}\left(p_{j \min }, p_{j \max }\right), j=1,2, \ldots, N$, with one generator as dependent generator.

2. Initialize the parent vector $p=\left[p_{1}, p_{2}, \ldots, p_{n}\right], i=$ $1,2, \ldots, N_{p}$ such that each element in the vector is determined by $p_{j} \sim \operatorname{random}\left(p_{j \min }, p_{j \max }\right), j=1,2, \ldots, N$, with one generator as dependent generator.

3. Calculate the overall objective function if the UCP is given in equation (3) using the trail vector $p_{i}$ and find the minimum of $F_{T i}$.

4. Initialize the parent vector $p=\left[p_{1}, p_{2}, \ldots, p_{n}\right], i=$ $1,2, \ldots, N_{p}$ such that each element in the vector is determined by $p_{j} \sim \operatorname{random}\left(p_{j \min }, p_{j \max }\right), j=1,2, \ldots, N$, with one generator as dependent generator.

5. Calculate the overall objective function if the UCP is given in equation (3) using the trail vector $p_{i}$ and find the minimum of $F_{T i}$. 


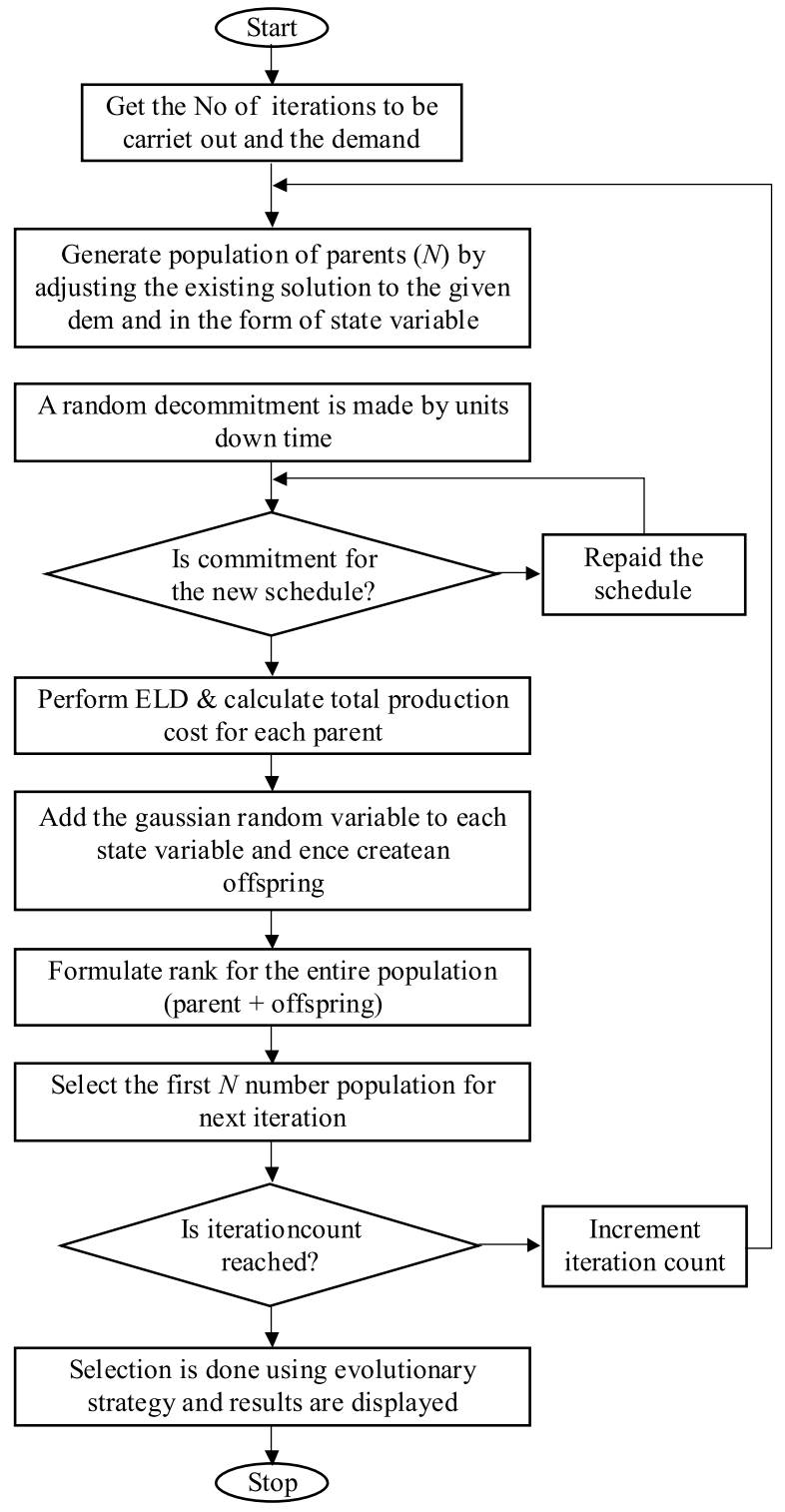

Fig. 2. Flowchart for EPTS for UCP

6. Create the offspring trail solution $p_{i}^{\prime}$ using the following steps.

(a) Calculate the standard deviation

$$
\sigma_{j}=\beta\left(F_{T i j} / \min \left(F_{T i}\right)\right)\left(P_{j \max }-P_{j \min }\right),
$$

(b) Add a Gaussian random variable $N\left(0, \sigma_{j}^{2}\right)$ to all the state variable of $p_{i}$, to get $p_{i}^{\prime}$.

7. Select the first $N_{p}$ individuals from the total $2 N_{p}$ individuals of both $p_{i} \& p_{i}^{\prime}$ using the following steps for next iteration.

(a) Evaluate $r=\left(2 N_{p} \operatorname{random}(0,1)+1\right)$,

(b) Evaluate each trail vector by $W_{p i}=\sum\left(W_{x}\right)$, where $x=1,2, \ldots, N_{p}, i=1,2, \ldots, 2 N_{p}$ such that $W_{x}=$ 1 if $F_{\text {Tij }} /\left(F_{\text {Tij }}+F_{\text {Tir }}\right)<\operatorname{random}(0,1)$, otherwise, $W_{x}=0$.

8. Sort the $W_{p i}$ in descending order and the first $N_{p}$ individuals will survive and are transcribed along with their elements to form the basis of the next generation.
9. The above procedure is repeated from step 2 until a maximum number of generations $N_{m}$ is reached.

10. Selection process is done using Evolutionary strategy.

\section{EVOLUTIONARY PROGRAMMING BASED TABU SEARCH FOR UCP}

\subsection{Tabu Search}

1. Take the parent as the initial feasible solution.

2. Take demand as control parameter and generate the trial solution.

3. Check for the stopping criterion.

4. If false, decrement system peak demand and go to step 2 .

5. If true, generate the optimal solution and calculate the total operating cost.

\subsection{EP based TS}

In the TS technique for solving UCP, Initial Operating Schedule status in terms of maximum real power generation of each unit is given as input. As we know that TS is used to improve any given status by avoiding entrapment in local minima, the offspring obtained from the EP algorithm is given as input to TS and the refined status is obtained. And Evolutionary Strategy selects the final status.

1. Get the demand for 24 hours and the number of iterations to be carried out.

2. Generate population of parents $(N)$ by adjusting the existing solution to the given demand to the form of state variables.

3. Unit down time makes a random recommitment.

4. Check for constraint in the new schedule by TS. If the constraints are not met then repair the schedule as given in Section 5-C.

5.Perform ELD and calculate total production cost for each parent.

6. Add the Gaussian random variable to each state variable and hence create an offspring. This will further undergo for some repair operations as given in Section 5-D. Following these, the new schedules are checked in order to verify that all constraints are met.

7. Improve the status of the evolved offspring and verify the constraints by TS.

8. Formulate the rank for the entire population.

9. Select the best $N$ number of population for next iteration.

10. Has iteration count reached? If yes go to step 11 else go to step 2.

11. Select the best population $(s)$ by Evolutionary strategy [29-31].

12. For the units, which are in the off states, calculate the cost for both cooling and banking.

13. Compare the cooling and banking costs, if banking cost is lesser than cooling, bank the unit.

14. Print the optimum schedule.

The flow chart for EPTS for UCP is shown in Fig. 2. 


\subsection{Repair Mechanism}

A repair mechanism to restore the feasibility of the constraints is applied and described as follows:

- Pick at random one of the OFF units at one of the violated hours.

- Apply the rules in section 3-C to switch the selected unit from $\mathrm{OFF}$ to $\mathrm{ON}$ keeping the feasibility of the down time constraints.

- Check for the reserve constraints at this hour. Otherwise repeat the process at the same hour for another unit.

\subsection{Making offspring feasible}

While solving the constrained optimization problem, there are various techniques to repair an infeasible solution [29-31]. In this paper we have chosen the technique, which evolve only the feasible solutions. That is the schedule, which satisfies the set of constraints as mentioned earlier. Here, in this paper, the selection routine is involved as "culling force" to eliminate the feasible schedules. Before the best solution is selected by evolutionary strategy, the trail is made to correct the unwanted mutations.

\section{NUMERICAL RESULTS}

A TPS in India with seven generating units, each with a capacity of $210 \mathrm{MW}$, has been considered as a case study. A time period of 24 hours is considered; the unit commitment problem is solved for these seven units and also compared. The required inputs for solving the UCP are briefed here. The total number of generating units, the maximum real power generation of each unit and the cost function parameters of each unit are tabulated for a day, respectively, as shown in Table 1 and Table 2 for TPS. The status of unit i at time $t$ and the start-up/shutdown status obtained are the necessary solution for TS, EP, EPTS, DP, LR methods for TPS. The comparison of the total costs and Central Processing unit (CPU) time is shown in Table 3 .

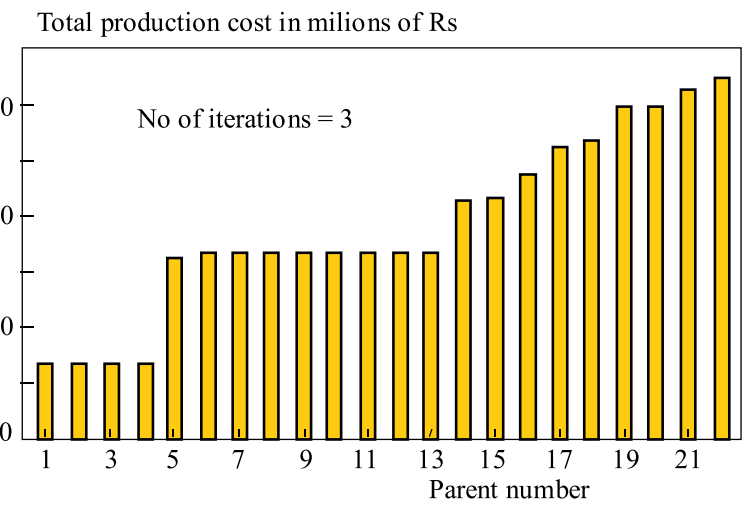

Fig. 3. Total production cost for 3 iterations

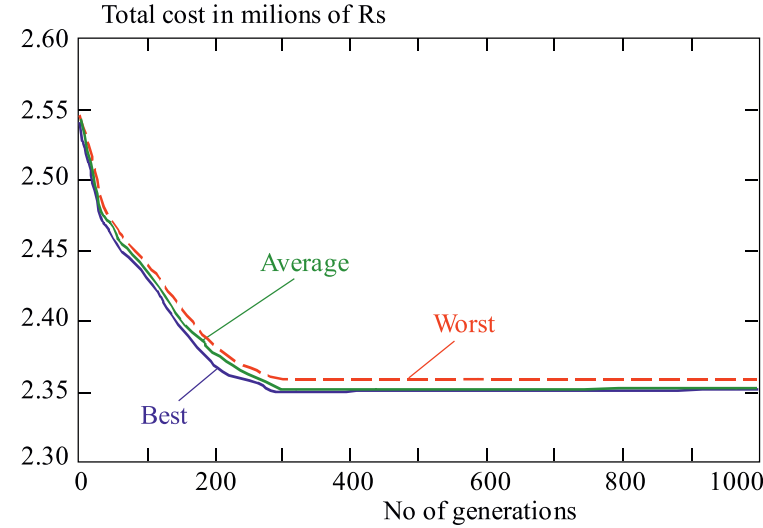

Fig. 4. EP average performance from 100 runs

Time in seconds Max.total production cost in milions of Rs

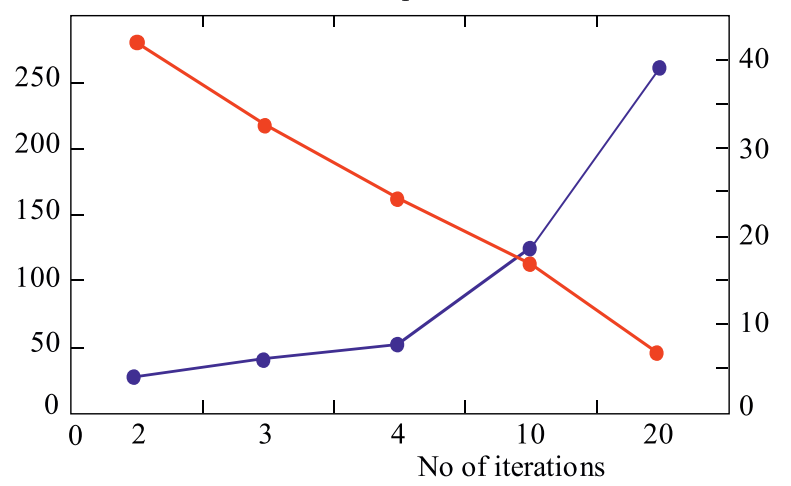

Fig. 5. No. of iterations vs time taken \& max. production cost

Figure 3 represents the total production cost obtained by each parent for three iterations in EP method. Similarly, for four and ten iterations are obtained. Figure 4 gives the plot of EP average performance from 100 runs. Figure 5 gives the plot of No. of iteration versus the time taken to complete those iterations and the maximum production cost obtained under each iteration. From these results, the EPTS method with cooling-banking constraints had lesser total cost and took lesser CPU time.

The proposed EPTS approach was compared to the related methods in the references indented to serve this purpose, such as the DP with a zoom feature, the SA, and the GA approaches. Further SA can start with any initial solution and improves on it to find optimal solution with a high probability. By means of stochastically searching multiple points at one time and considering trail solutions of successive generations, the EPTS approach avoids entrapping in local optimum solutions. Also, disadvantages of huge memory size required by the TS method are eliminated. Moreover, intellectual schemes of encoding and decoding entailed by the GA approach are not needed in the proposed EPTS approach. The problem of power unbalance previously existing in the solution of GA is circumvented as well in this paper. In comparison with the results produced by the referenced techniques, the EPTS method obviously displays a satisfactory performance with respect to the quality of its evolved solutions and to its computational requirements. 
Table 1. Daily Generation of Seven Units in MW

\begin{tabular}{ccccccccc}
\hline Hour & Pmax & I & II & III & IV & V & VI & VII \\
\hline 1 & 840 & 60 & 80 & 100 & 101 & 149 & 150 & 200 \\
2 & 757 & 60 & 0 & 100 & 100 & 147 & 150 & 200 \\
3 & 775 & 60 & 0 & 100 & 115 & 150 & 150 & 200 \\
4 & 773 & 60 & 0 & 100 & 113 & 150 & 150 & 200 \\
5 & 770 & 60 & 0 & 100 & 110 & 150 & 150 & 200 \\
6 & 778 & 60 & 0 & 100 & 118 & 150 & 150 & 200 \\
7 & 757 & 60 & 0 & 100 & 100 & 147 & 150 & 200 \\
8 & 778 & 60 & 0 & 100 & 118 & 150 & 150 & 200 \\
9 & 770 & 60 & 0 & 100 & 110 & 150 & 150 & 200 \\
10 & 764 & 60 & 0 & 100 & 104 & 150 & 150 & 200 \\
11 & 598 & 60 & 0 & 99 & 97 & 142 & 0 & 200 \\
12 & 595 & 60 & 0 & 100 & 96 & 139 & 0 & 200 \\
13 & 545 & 0 & 0 & 100 & 99 & 146 & 0 & 200 \\
14 & 538 & 0 & 0 & 99 & 97 & 142 & 0 & 200 \\
15 & 535 & 0 & 0 & 100 & 96 & 139 & 0 & 200 \\
16 & 466 & 0 & 0 & 0 & 116 & 150 & 0 & 200 \\
17 & 449 & 0 & 0 & 0 & 101 & 148 & 0 & 200 \\
18 & 439 & 0 & 0 & 0 & 97 & 142 & 0 & 200 \\
19 & 466 & 0 & 0 & 0 & 116 & 150 & 0 & 200 \\
20 & 463 & 0 & 0 & 0 & 113 & 150 & 0 & 200 \\
21 & 460 & 0 & 0 & 0 & 110 & 150 & 0 & 200 \\
22 & 434 & 0 & 0 & 0 & 95 & 139 & 0 & 200 \\
23 & 530 & 60 & 0 & 0 & 120 & 150 & 0 & 200 \\
24 & 840 & 60 & 80 & 100 & 101 & 149 & 150 & 200 \\
\hline & & & & & & & &
\end{tabular}

Table 2. Generation System Operation Data

\begin{tabular}{ccccccccc}
\hline & \multicolumn{1}{c}{ Running Cost } & \multicolumn{3}{c}{ Start-up Cost } \\
Unit & $\mathrm{P}_{\min }$ & $\mathrm{P}_{\max }$ & $\mathrm{C}_{i}$ & $\mathrm{~B}_{i}$ & $\mathrm{~A}_{i}$ & $\mathrm{So}_{i}$ & $\mathrm{D}_{i}$ & $\mathrm{E}_{i}(\mathrm{Rs})$ \\
& $(\mathrm{MW})$ & $(\mathrm{MW})$ & $(\mathrm{Rs})$ & $(\mathrm{Rs} /$ & $(\mathrm{Rs} /)$ & $(\mathrm{Rs})$ & $(\mathrm{Rs})$ & $(\mathrm{Rs})$ \\
& \multicolumn{9}{c}{$(\mathrm{MWh})$} & $\left.\mathrm{MWh}^{2}\right)$ & & & \\
\hline 1 & 15 & 60 & 750 & 70 & 0.255 & 4250 & 29.5 & 10 \\
2 & 20 & 80 & 1250 & 75 & 0.198 & 5050 & 29.5 & 10 \\
3 & 30 & 100 & 2000 & 70 & 0.198 & 5700 & 28.5 & 10 \\
4 & 25 & 120 & 1600 & 70 & 0.191 & 4700 & 32.5 & 9 \\
5 & 50 & 150 & 1450 & 75 & 0.106 & 5650 & 32 & 9 \\
6 & 50 & 150 & 4950 & 65 & 0.068 & 14100 & 37.5 & 4.5 \\
7 & 75 & 200 & 4100 & 60 & 0.074 & 11350 & 32 & 5.5 \\
\hline
\end{tabular}

\section{CONCLUSION}

This paper presents an EP based TS method with cooling-banking constraints the unit commitment problem. In this method, the essential processes simulated in the procedure are mutation, competition, and selection. The mutation rate is computed as a function of the ratio of the total cost by the schedule of interest to the cost of the best schedule in the current population. Competition and selection are applied to select from among the parents and the offspring, the best solutions to form the basis of the subsequent generation. In this proposed work, the parents are obtained from a pre-defined set of solution's ie each and every solution is obtained from the $\mathrm{SA}$ method. Then, a random recommitment is carried out with respect to the unit minimum down times, and the selection process is done using Evolutionary Strategy.

In comparison with the results produced by the referenced techniques (EP, DP, LR and TS), the EPTS method obviously displays a satisfactory performance. There is no obvious limitation on the size of the problem that must be addressed, for its data structure is such that the search space is reduced to a minimum; No relaxation of constraints is required; instead, populations of feasible solutions are produced at each generation and throughout the evolution process; Multiple near optimal solutions to the problem involving multiple constraints and conflicting objectives can be obtained in a reasonable time with the use of heuristics; It works only with feasible solutions generated based on heuristics, thus avoiding the computational burden entailed by the GA methods which first generate all feasible solutions and then purge the infeasible ones.

Table 3. Comparisons of cost and CPU time

\begin{tabular}{|c|c|c|c|}
\hline System & Methods & $\begin{array}{l}\text { Total } \\
\text { Cost } \\
(\mathrm{pu})\end{array}$ & $\begin{array}{l}\text { CPU } \\
\text { Time } \\
\text { (Sec) }\end{array}$ \\
\hline \multirow{6}{*}{$\begin{array}{c}7 \text { Unit } \\
\text { (Practical) }\end{array}$} & $\mathrm{DP}$ & 1.00000 & 130 \\
\hline & LR & 0.97843 & 115 \\
\hline & TS & 0.94580 & 80 \\
\hline & $\mathrm{EP}$ & 0.93461 & 66 \\
\hline & $\begin{array}{l}\text { EPTS (Without } \\
\text { Cooling \& Banking) }\end{array}$ & 0.92392 & 56 \\
\hline & $\begin{array}{l}\text { EPTS (With } \\
\text { Cooling \& Banking) }\end{array}$ & 0.92056 & 54 \\
\hline \multirow{6}{*}{$\begin{array}{l}10 \text { Unit } \\
\text { (IEEE) }\end{array}$} & $\mathrm{DP}$ & 1.00000 & 260 \\
\hline & LR & 0.97183 & 235 \\
\hline & TS & 0.94222 & 200 \\
\hline & $\mathrm{EP}$ & 0.94071 & 186 \\
\hline & $\begin{array}{l}\text { EPTS (Without } \\
\text { Cooling \& Banking) }\end{array}$ & 0.92090 & 176 \\
\hline & $\begin{array}{l}\text { EPTS (With } \\
\text { Cooling \& Banking) }\end{array}$ & 0.91710 & 172 \\
\hline \multirow{6}{*}{$\begin{array}{l}26 \text { Unit } \\
\text { (IEEE) }\end{array}$} & DP & 1.00000 & 1878 \\
\hline & $\mathrm{LR}$ & 0.96642 & 1860 \\
\hline & TS & 0.93706 & 1810 \\
\hline & $\mathrm{EP}$ & 0.92814 & 1785 \\
\hline & $\begin{array}{l}\text { EPTS (Without } \\
\text { Cooling \& Banking) }\end{array}$ & 0.90900 & 1774 \\
\hline & $\begin{array}{l}\text { EPTS (With } \\
\text { Cooling \& Banking) }\end{array}$ & 0.90600 & 1766 \\
\hline \multirow{6}{*}{$\begin{array}{l}34 \text { Unit } \\
\text { (IEEE) }\end{array}$} & $\mathrm{DP}$ & 1.00000 & 6865 \\
\hline & LR & 0.96197 & 6824 \\
\hline & TS & 0.93300 & 6800 \\
\hline & $\mathrm{EP}$ & 0.92400 & 6792 \\
\hline & $\begin{array}{l}\text { EPTS (Without } \\
\text { Cooling \& Banking) }\end{array}$ & 0.90718 & 6772 \\
\hline & $\begin{array}{l}\text { EPTS (With } \\
\text { Cooling \& Banking) }\end{array}$ & 0.90425 & 6764 \\
\hline
\end{tabular}




\section{REFERENCES}

[1] SNYDER, W. L.-POWELL, H. D.-RAYBURN, C.: Dynamic Programming approach to Unit Commitment, IEEE Trans. Power Systems 3 (1987), 339-350.

[2] HSU, Y. Y.-LIANG, C. C.-LIN, C. J.-HUANG, C. T.: Dynamic Security Constrained Multi-Area Unit Commitment, IEEE Trans. Power Systems 6 (1991), 1049-1055.

[3] TAKRITI, S.-BIRGE, J. R.: Using Integer Programming To Refine Lagrangian-Based Unit Commitment Solutions, IEEE Trans. Power Systems 15 (2000), 151-156.

[4] MOHAN, M. R.-KUPPUSAMY, K.-KHAN, A. M.: Optimal Short-term Hydro-Thermal Scheduling using Decomposition Approach and Linear Programming Method, International Journal of Electrical Power and Energy Systems 14 (1992), $39-44$.

[5] NGUNDAM, J. M.-KEnFACK, F.-TATIETSE, T. T. : Optimal Scheduling of Large-Scale Hydrothermal Power Systems Using the Lagrangian Relaxation Technique, International Journal of Electrical Power \& Energy Systems 22 (2002), 237-245.

[6] BAKIRTZIS, A. G.-ZOUMAS, C. E. : Lambda of Lagrangian Relaxation Solution to Unit Commitment Problem, IEE Proc. Generation, Transmission and Distribution 147 (2000), 131-136.

[7] CHANG, C. P.-LIU, C. W.-LIU, C. C. : Unit Commitment by Legrangian Relaxation and Genetic Algorithms, IEEE Trans. Power Systems 15 No. 2 (2000), 707-714.

[8] NIEVA, R.-INDA, A.-GUILLEN, I. : Lagrangian Reduction of Search Range for Large Scale Unit Commitment, IEEE Trans. Power Systems 2 (1987), 465-473.

[9] COHEN, A. I.-YOSHIMURA, M. : A Branch and Bound Algorithm for Unit Commitment, IEEE Trans. Power and Apparatus 102 (1983), 444-451.

[10] OUYANG, Z.-SHAhideHPOUR, S. M. : Short Term Unit Commitment Expert System, International Journal of Electrical Power System Research 20, 1-13.

[11] SU, C. C.-HSU, Y. Y.: Fuzzy Dynamic Programming: an Application to Unit Commitment, IEEE Trans. Power Systems 6 (1991), 1231-1237.

[12] SASAKI, H.-WATANABE, M.-KUBOKAWA, J.-YORINO, N.: A Solution Method of Unit Commitment by Artificial Neural Networks, IEEE Trans. Power Systems 7 (1992), 974-981.

[13] PADHY, N. P.: Unit Commitment using Hybrid Models: A Comparative Study for Dynamic Programming, Expert Systems, Fuzzy System and Genetic Algorithms, International Journal of Electrical Power \& Energy Systems 23 (2000), 827-836.

[14] ZHUANG. F.-GALIANA, F. D. : Unit Commitment by Simulated Annealing, IEEE Trans. Power Systems 5 (1990), 311-318.

[15] KIRKPATRICK, S.-GELATT, C. D.—VECEHI, M. P.Jr.: Optimisation by SA, Science 220 (1983), 4598.

[16] SHOKRI, Z. S.-ALSUlTAN, K. : A Simulated Annealing Algorithm for the Clustering Problem, Pattern Recognition 24 (1991), 1003-1008.

[17] MANTAWY, A. H.-YOUSSEF, L.-ABDEL-MAGID,-SHOKRI, Z. S. : A Simulated Annealing Algorithm for Unit Commitment, IEEE Trans. Power Systems 13 (1998), 197-204.

[18] MANTAWY, A. H.-YOUSSEF, L.-ABDEL-MAGID-SHOKRI, Z. S. : A Unit Commitment by Tabu Search, IEE Proc. Generation, Transmission and Distribution 145 (1998), 56-64.
[19] LIN, W. M.-CHENG, F. S.-TSAY, M. T.: An Improved Tabu Search for Economic Dispatch With Multiple Minima, IEEE Trans. Power Systems 17 (2002), 108-112.

[20] BAI, X.-SHAHIDEHPOUR, M.: Hydro-Thermal Scheduling by Tabu Search and Decomposition Method, IEEE Trans. Power Systems 11 (1996), 968-975.

[21] WU, Y. G.-HO, C. Y.-WANG, D. Y.: A Diploid Genetic Approach to Short-Term Scheduling of Hydro-Thermal System, IEEE Trans. Power Systems 15 (2000), 1268-1274.

22] HONG, Y. Y.-LI, C. Y.: Genetic Algorithm Based Economic Dispatch for Cogeneration Units Considering Multiplant Multibuyer Wheeling, IEEE Trans. Power Systems 17 (2002), $134-140$.

23] MANTAWY, A. H.-YOUSSEF, L. A. M.-SHOKRI, Z. S. : Integrating Genetic Algorithm, Tabu Search and Simulated Annealing for the Unit Commitment Problem, IEEE Tans. Power Systems 14 (1999), 829-836.

24] WONG, K. P. S.-WONG, Y. W.: Combined Genetic Algorithm/Simulated Annealing/Fuzzy Set Approach to Short-Term Generation Scheduling with Take-or-pay Fuel Contract, IEEE Trans. Power Systems 11 (1996), 128-135.

25] JUSTE, K. A.-KITA, H.-TANAKA, E.-HASEGAWA, J. : An Evolutionary Programming Solution to the Unit Commitment Problem, IEEE Trans. Power Systems 14 (1999), 1452-1459.

[26] YANG, H. T.-YANG, P. C.-HUANG, C. L.: Evolutionary Programming Based Economic Dispatch for Units with Non-smooth Fuel Cost Functions, IEEE Trans. Power Systems 11 (1996), 112-117.

[27] WOOD, A. J.-WOOLEnBerG, B. F. : Power Generation and Control, $2^{\text {nd }}$ Edn., John Wiley and Sons, New York, 1996.

[28] ARTS, E.-KORST, J.: A Stochastic Approach to Combinatorial Optimization and Neural Computing, John Wiley \& Sons Ltd, New York, 1989.

[29] FOGEL, D. B. : Evolutionary Computation, Toward a New Philosophy of Machine Intelligence, IEEE Press, New York, 1995.

[30] BACK, T.: Evolutionary Algorithms in Theory and Practice, Oxford University Press, New York, 1996.

30] FOGEL, L. J.-OWENS, A. J.-WALSH, M. J.: Artificial Intelligence through Simulated Evolution, John Wiley \& Sons, inc, New York, 1996.

Received 29 January 2010

C. Christober Asir Rajan born on 1970 and received his BE (Distinguished) degree (Electrical and Electronics) and ME (Distinguished) degree (Power System) from the Madurai Kamaraj University (1991 and 1996), Madurai, India. And he received his postgraduate degree in DIS (Distinguished) from the Annamalai University, Chidambaram. He did PhD in Power System at College of Engineering, Guindy, Anna University (2001-2004), Chennai, India. He published technical papers in International and National Journals and Conferences. He is currently working as Associate Professor in Electrical Engineering Department at Pondicherry Engineering College, Pondicherry, India. His area of interest is power system optimization, operational planning and control. He acquired Member in ISTE and MIE in India. 